\title{
A Contemporary View on Felix Pinkus' Concept of the Vitreous Membrane
}

\author{
Karola Stieler Annika Vogt Wolfram Sterry Norbert Haas \\ Ulrike Blume-Peytavi \\ Department of Dermatology and Allergology, Clinical Research Center for Hair and Skin Science, Charité - \\ Universitätsmedizin Berlin, Corporate Member of Freie Universität Berlin, Humboldt-Universität zu Berlin, and \\ Berlin Institute of Health, Berlin, Germany
}

\section{Keywords}

Pinkus - Vitreous membrane - Basement membrane ·

Hair follicle

\begin{abstract}
Introduction: Felix Pinkus' concept of the vitreous membrane (VM) published in 1927 describes circular folds protruding into the outer root sheath (ORS), which, in his opinion, serve as interdigitations between the outer root sheath (ORS) and the VM. This concept currently seems to have fallen into oblivion. Objective: To determine the origin and possible function of the VM in the proliferation and vascularization of the hair follicle (HF). Methods: Serial investigation of healthy skin probes with histological (hematoxylin \& eosin and periodic acid-Schiff) and immunohistochemical examination (Ki67, CD56, CD8, and collagen IV) were performed. Results: Morphological variations of the VM in various HFs such as protrusions and folds, the latter unilateral, bilateral or circular, some acute-angled, were found. Similarly, protrusions of the VM into the ORS were observed, that consisted of capillary tissue together with perifollicular tissue and VM mimicking minimal variants of the dermal papilla. Conclusions: Pinkus' concept of the VM is revisited, reproduced and
\end{abstract}

\section{KARGER}

(c) 2019 S. Karger AG, Basel

E-Mail karger@karger.com

www.karger.com/sad possible functions are proposed. Since these structures are found in a HF region with a high metabolic dynamism, they may be involved in differentiation or nutrition, or else be formed as a result of pressure arising from outgrowing hair shafts.

(c) 2019 S. Karger AG, Basel

\section{Introduction}

The vitreous (glassy, basement, or hyaline) membrane (VM) around the outer part of the hair follicle (HF) [1] separates the follicular outer root sheath (ORS) from its adjacent fibrous sheath as a connective tissue-epithelial junction [2]. In the contemporary literature, collagen IVpositive VM [3,4] is described as a noncellular layer detectable by light microscopy that invests the entire HF. During the hair cycle, it undergoes characteristic morphological changes (Table 1). It is known that the ultrastructure of the VM comprises 3 separate elements. On the inside, there is a tangle of argyrophilic fibrils interdigitating with serrations of the ORS cells; just peripheral to this layer is a thin but distinct non-stainable (with periodic acid-Schiff [PAS]) membrane followed by the thick 
PAS-positive hyaline membrane [5]. In the current literature, the VM is mostly depicted as a smooth, uniform structure. In contrast, Pinkus' concept is that the VM has folds that merge deep into the ORS and can reach the same thickness as the glassy membrane itself [6, p. 128]. He interpreted these folds as annular, shelf-like appendices (Fig. 1a, b) [6, pp. 128, 192].

This study aims to investigate the validity of Pinkus' concept and search for these structures in normal HFbearing skin.

\section{Materials and Methods}

Routine histology from 11 human scalp and beard samples obtained from otherwise healthy patients ( 2 males and 9 females aged 30-73 years) undergoing routine surgery at our department for basal cell carcinoma, dysplastic nevus, actinic keratosis, pseudopelade Brocq, and androgenic alopecia were examined histologically.

These samples, taken from the healthy margins of the biopsy specimen, were paraffin-embedded, cut into $6-\mu \mathrm{m}$ slices, and stained with hematoxylin \& eosin (HE) and PAS. The skin from 1 person was additionally stained for Ki67, CD56, CD8, and collagen IV (Table 2). The stained slides were compared to Pinkus' paintings and sketches. To observe the distribution of agyrophilic nerve fibers in these variations of the VM, Gomori staining was used on slides from 2 different patients.

\section{Staining Methods}

Paraffin-embedded slides were pretreated with xylene and alcohol in decreasing concentrations routinely. Avidin-streptavidin staining [7] and the alkaline phosphatase (AP) anti-AP (APAAP) technique [8] were accomplished using conventional protocols.

\section{Results}

\section{Variations in Morphology of the VM}

In all 11 patients, we found folds that consisted entirely of VM material and protrusions composed of VM plus connective tissue and capillaries. These variations were all found in the transitional zone, i.e., the hair cycle-dependent part of the hair follicle. In the "2-dimensional" slides, there were 3 types of fold: (i) unilateral, i.e., only on 1 side of the hair follicle, (ii) bilateral, and (iii) circular. Unilateral folds were found in 4 of 7 investigated specimens (Fig. 2a). These reached a maximal length of $171 \mu \mathrm{m}$ and a thickness of 2-3 $\mu \mathrm{m}$. In 2 of these 7, the folds extended deep into the ORS in the opposite direction to the hair growth, with a thickening of the ORS in the affected area (Fig. 2a, b).
Table 1. Morphology of the vitreous membrane in relation to hair follicle (HF) growth [1]

\begin{tabular}{|c|c|c|}
\hline Hair cycle stage & Proximal part of HF & Distal part of HF \\
\hline Early anagen & thin, inconspicuous & thin \\
\hline Late anagen & & $500-1,000 \AA$ \\
\hline Early catagen & & thick \\
\hline Later catagen & thin, inconspicuous & highly pleated, thick \\
\hline Telogen & unchanged & resorbed \\
\hline
\end{tabular}

In 2 of 7 biopsies, a bilateral fold was seen. Two patients showed circular folds (Fig. 2c); 1 had the folds at regular intervals, which resulted in a 2-dimensional rope-ladder pattern (Fig. 2d). The folds consisted of clear duplications of the innermost PAS-positive layer of the VM with only a sparse or absent middle layer (in $3 / 7$ ) (Fig. 2c[i]). In 4 out of 7, duplication was not clearly present (Fig. 2d, e). Only 1 fold was connected to the inner root sheath (IRS; not shown). In one HF, a cystic structure was seen in direct contact with the fold (Fig. 2e).

Cone-like protrusions of the ORS were seen near to large folds in 2 of 7 specimens (Fig. 2a, b). No capillaries were found in these folds in the basement membrane of the dermoepidermal junction.

Protrusions of the VM were seen in 8 cases, each containing a capillary (Fig. 3a, b). A pectinated, inlaying layer of fibrils of the VM was found on most of the protrusions, but not any of the folds (Fig. 3c). All protrusions had 1 capillary inside like in the papillary dermis of the skin.

\section{Staining Patterns}

The folds and protrusions stained positive for PAS and Gomori and showed a continuity of the staining patterns from the VM to the folds. Alcian blue was negative in the cystic formation in the ORS. The collagen IV marker for VM stained positive in all the folds and protrusions investigated, specifically the innermost part of the VM (i.e., the fibrils) and its outer layer. Interestingly, the marker revealed a nonstainable layer of internal fibrils that emanated from the outer layer and penetrated through the middle and innermost layers (Fig. 3d). Protrusions of the VM into the HF showed the innermost membrane with its pectinate fibrils positive, the middle negative, and the outer layer positive for collagen IV (Fig. 3c).

Detection of Ki67 and CD56 (NCAM) was not increased within the ORS folds and protrusions. The surrounding ORS of the folds and protrusions was negative 
Table 2. Antibodies used for immunohistochemistry

\begin{tabular}{|c|c|c|c|c|c|}
\hline Antibody (clone) & Specificity & Source & Dilution & Target retrieval & Method \\
\hline Ki67 & proliferation in outer root sheath cells [15] & Dako & $1: 50$ & citrate buffer pH6 & peroxidase \\
\hline CD8/CK15 & hair follicle stem cells $[16-18]$ & Dako & $1: 100$ & citrate buffer $\mathrm{pH} 6$ & peroxidase \\
\hline CD31 & endothelium $[19,20]$ & Dako & $1: 50$ & citrate buffer $\mathrm{pH} 6$ & peroxidase \\
\hline CD34 & marker of hair follicle bulge keratinocytes $[21,22]$ & Immunotech & $1: 300$ & citrate buffer pH6 & peroxidase \\
\hline CD56 & bulge region [23] & Zymed & $1: 400$ & EDTA pH8 & peroxidase \\
\hline
\end{tabular}

Fig. 1. a Pinkus' original detailed drawings (ink with watercolor; $6 \times 15 \mathrm{~cm}$ ). Longitudinal cut through the transitory part of the hair follicle. The black arrow shows the outer root sheath (ORS). The white arrow shows the folds of the vitreous membrane (VM), resembling a rope-ladder that pervades the ORS. b Pinkus' original pencil drawing $(7 \times 10 \mathrm{~cm})$. Three-dimensional representation of his concept with the circular folds of the VM protruding into the ORS.
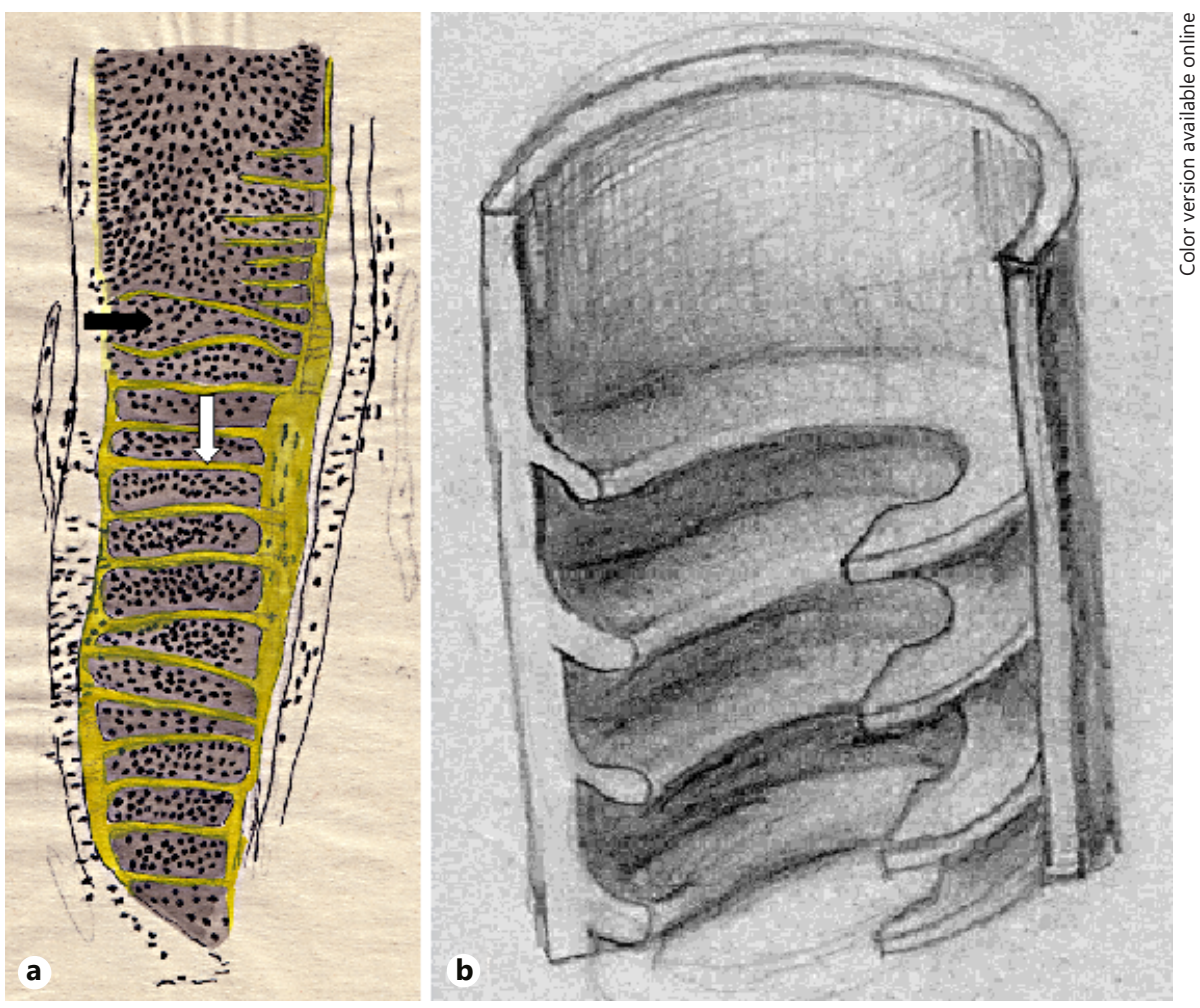

for CD34, CD8, and CK15. There was no distinctive presence of CK14 in the abovementioned areas. CD31 was negative within the capillaries of the protrusions.

\section{Discussion/Conclusion}

Felix Pinkus (1868-1947), who discovered the so-called "Haarscheibe," (the Pinkus corpuscule) [9], was one of the most celebrated masters of cutaneous histology and published his knowledge of the HF apparatus in several books and articles. He also produced his own graphs and illustrations for his publications including his masterpiece, the 378-page article "Die normale Anatomie der Haut" in Jadassohn's "Handbuch der Haut- und Geschlechtskrankheiten" in 1927 [6]. Recently, so far unknown drawings of the hair apparatus by Felix Pinkus have been brought to light. These were found among articles and papers which had belonged to Felix Pinkus. They were donated by his grandson Walter Pinkus and are currently exhibited in the "Pinkus library" at our Department of Dermatology.

We found the drawings presented here filed randomly in different folders, without any explanatory text attached and sparse-to-absent inscription. We investigated the literature to ascertain whether Pinkus had ever used these drawings in his publications but could find no evidence 


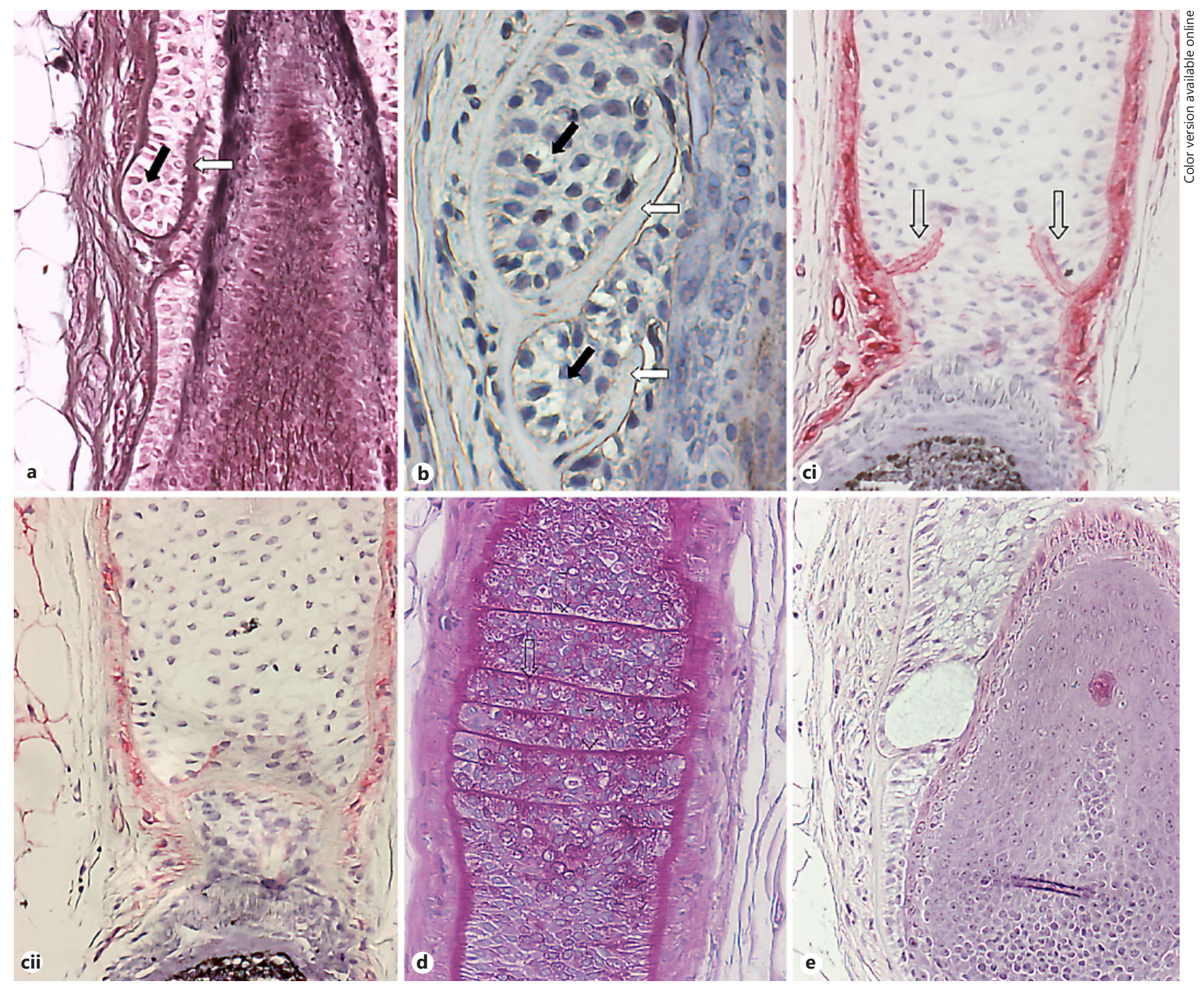

Fig. 2. Our histological investigation of human scalp skin. a Unilateral fold extends deeply into the outer root sheath (ORS). Matrix at the right side. The black arrow shows the conelike protrusion of the ORS. The white arrow shows the fold of the vitreous membrane $(\mathrm{VM})$. PAS. $\times 200$. b Cone-like thickened ORS areas (black arrows) and 2 acute-angled folds (white arrows) without increased proliferation rate. Matrix cells of the inner root sheath (right). Fibrous sheath with capillaries (left).

thereof. They represent cross-sections of HFs at different magnifications; their focus is the VM and they seem to illustrate his concept of the glassy membrane.

\section{Pinkus' Concept of the VM}

"The vitreous membrane is a hyaline, non-textured layer whose visibility is dependent on the thickness of the hair
Ki67. $\times 400$. c(i) Circular folds in the suprabulbar region (transparent arrows). Doubled VM. Collagen IV. $\times 200$. c(ii) Serial section of (i) with a continuous fold. d Circular folds pervading the ORS resemble a rope-ladder as demonstrated in Pinkus' original detailed drawing (Fig. 1a). Bilateral VM and fibrous sheath. Transitory part of the hair follicle, anagen. PAS. $\times 200$. e Cystic formation within ORS at the site of the fold (area indicated by black lines). HE. $\times 200$. 
form the basis of our description can be reconstructed by putting together information gleaned from serial slides" [6, p. 128f]. Pinkus regarded the VM as a kind of "sliding layer," between the fibrous sheath and the ORS, which is subjected to constant metabolic activity [6, p. 131ff].

\section{Current Knowledge of the VM Histology Concerning the HF Growth Phase}

The transitory part of the HF (below the isthmus) is a dynamic zone involved in the construction, deconstruction, and movement of the hair shaft towards the skin surface. The VM that surrounds the entire HF appears thin and inconspicuous in early anagen and telogen. In early catagen, when large numbers of cells of the HF undergo disintegration, this PAS-positive hyaline membrane around the lower part of the follicle and the dermal papilla reaches a thickness of about 500-1,000 A. When, in later catagen, apoptotic HF cells are absorbed, the VM remains without support and consequently becomes pleated. Hence, during the last stages of catagen, the highly pleated glassy membrane encloses the atrophying cells of the lower part of the follicle. At telogen, the thick and pleated glassy membrane around the lower hair follicle becomes fragmented and absorbed [10, p. 89]. Around the upper part of the HF, which does not undergo any cyclic changes, the basal lamina remains unchanged [11].

These hair cycle-dependent changes are well known and often illustrated. However, the morphological variant, the circular folds described by Pinkus, are, to our knowledge, not mentioned in contemporary literature and have probably fallen into oblivion. In the literature, the folds are depicted [12] but not annotated. No comments on their possible origin and function have been found. Here, we present Pinkus' old painted findings of the VM depicted in contemporary 2-dimensional histological slides that show unilateral and bilateral folds; extrapolated into a 3-dimensional structure, these slides show circular folds. Additionally, protrusions of the VM into ORS are shown. To speculate on the origin, function, and significance of these folds and protrusions, we tried to answer the following questions.

\section{Do the Folds Extend into the ORS or Does the ORS \\ Protrude into the VM?}

On the basis of the observation that the ORS is partly cone-like and thickened in areas with large folds, reminiscent of the so-called "bulge activation hypothesis" [13, 14], specimens were stained to find evidence of stem cells in this region, but the result was negative. Since no increased proliferation of Ki67 was found in these "cones"

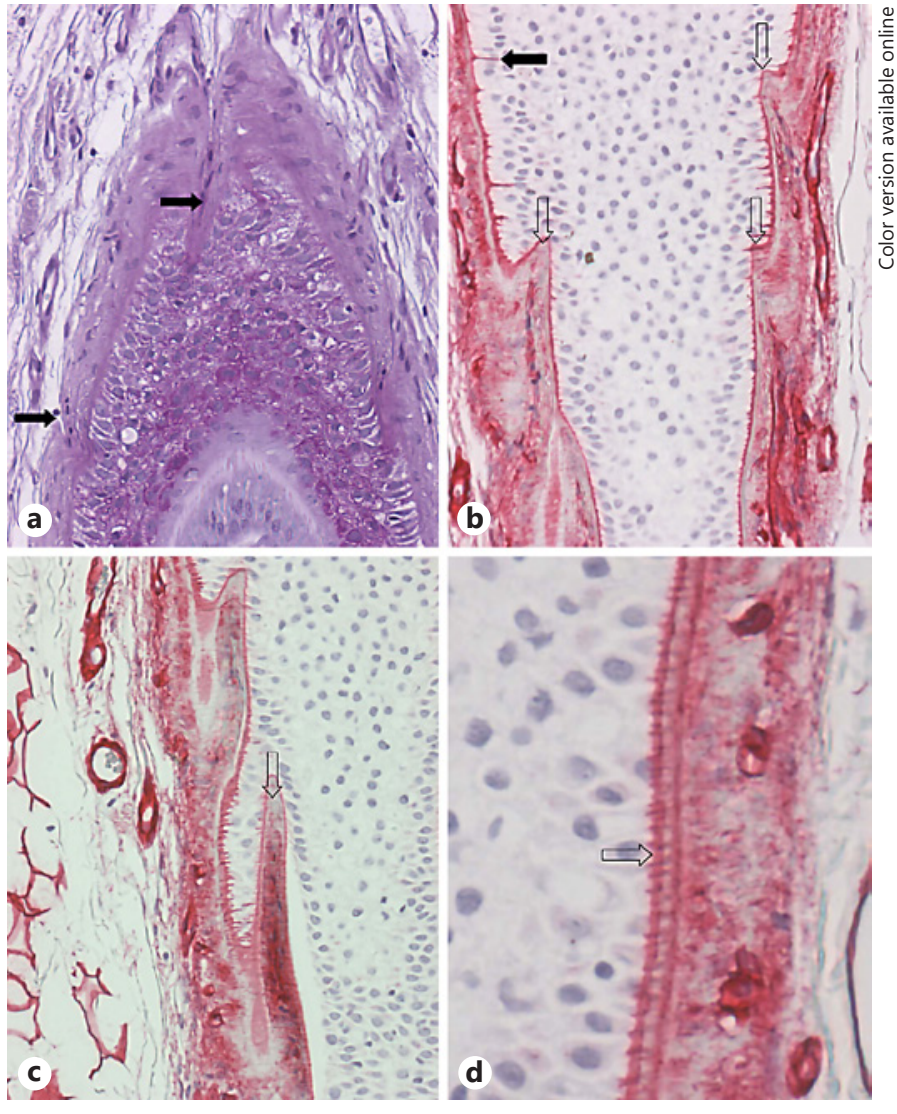

Fig. 3. a Protrusion with a capillary (black arrow) protruding deep into the suprabulbar outer root sheath (ORS), anagen. PAS. $\times 200$. b Protrusions (transparent arrows) lying opposite one another, with long pectinated fibrils (black arrow). Collagen IV. $\times 200$. c Protrusion (transparent arrow) with pectinated fibrils. Collagen IV. $\times 200$. d VM (transparent arrow) composed of a collagen IVpositive inner and outer layer, with a nonstainable layer in between and fibrils attached to the outer layer. ORS (left). Fibrous sheath (right). Collagen IV. Cutout from original magnification $(\times 200)$.

of the ORS, in our opinion, it is implausible that the folds would result from excessive growth of the ORS towards the fibrous sheath.

\section{Do the Folds Really Belong to the VM?}

Based on our histochemical findings, it was evident that the folds formed part of the VM, because their staining with PAS, collagen IV, and silver staining was congruent to the VM.

\section{Is There a Concertina-Like Effect during the Hair Cycle?}

During anagen, the ORS is surrounded by the VM, and the VM by the fibrous sheath. In the bulb region, the hyaline membrane is continuous with the papillary basement 
membrane surrounding the papilla; the latter, in turn, is continuous with the dermal sheath. For simplification, we regarded these components as 1 continuous unit.

It has already been shown that dermal-sheath cells can travel into the dermal papilla to increase the hair shaft diameter or contrawise [3]. This unit expands and contracts when the newly developing anagen hair descends all the way down to the base of the follicle and subcutaneous tissue; it then starts retreating upwards during catagen and goes all the way up to the level of the bulge in telogen. The hair does not always return to its original position in the subcutaneous tissue where it resumes anagen.

By having pleats and folds, the hyaline membrane has the opportunity to stretch out to accommodate the growing anagen hair and then contract down again in telogen. To visualize the unit's movement, it can be compared to a concertina. When it is fully extended downwards to its anagen position, only pleats are present. However, when it goes into catagen, the apoptotic ORS with the contracted hair root leaves the convoluted hyaline membrane behind, as a thickened and plicated mass in the bulge region that is normally resorbed in telogen. When the HF undergoes a shortened telogen phase and hair growth is induced prematurely (before the VM is absorbed), the new downward growing HF is surrounded by the "old" VM, or at least parts of it. It thus defoliates the VM like a concertina. If the growth of the new HF does not conform to the last phase, the concertina-like effect could explain the folds described above as being due to incomplete stretching of the VM. The detection of folds in a neighboring HF in the same growth stage confirmed that this seldom-observed phenomenon in routine histology is not sporadic. The cyst might be due to an incorrect development in this process (Fig. 2e).

\section{Why Are Folds Acute-Angled?}

The partly emerging acute angle towards the ostium of the HF may be formed by incomplete defoliation of the old VM and the direction of growth of the ORS towards the fatty tissue in anagen, resulting in a dragging of the fold behind the ORS cells. In contrast, the pectinated fibers of the VM have always been found to be rectangular.

\section{The Rope-Ladder Pattern}

The rope-ladder pattern (Fig. 1a, 2d) depicted by Pinkus) may be explained by the presence of the tangential section on the external edge of the ORS. The bilateral folds (Fig. 2c) suppose the idea of circular formation. The unilateral folds can be explained as an incomplete formation or a helical arrangement.
The Possible Function of the Protrusions in Nutrition

Like the epidermis, the entire follicular epithelium lacks blood vessels; for this reason, it is nourished by diffusion through the VM. Messenger et al. [12] have found evidence for bilaterally orientated longitudinal blood vessels around the transitory part of the HF. We found evidence of an adjacent network of capillaries, single capillary loops of which invaginate into the ORS in the form of protrusions. In doing so, these are accompanied by perifollicular tissue and a coating of VM, which is known as a basement membrane. The protrusions resemble, in principle, the composition of a dermal papilla. Like the papillary dermis, these protrusions of perifollicular fibrous tissue enlarge the VM's surface on the one hand and the neighboring epithelium on the other, thereby forming an enlarged contact area which facilitates a greater exchange of nutrients.

\section{Acknowledgements}

This paper is dedicated to Dr. David A. Whiting and his memory. The authors gratefully acknowledge Dr. David A. Whiting for the fruitful and stimulating discussion about the histological slides and our assumptions.

\section{Statement of Ethics}

The Ethics Committee of the Charité - Universitätsmedizin Berlin approved the research protocol 23-11-2003 and the study was performed according to the Good Clinical Practice guidelines. Written informed consent was obtained.

\section{Disclosure Statement}

All authors declare that they have no conflict of interest.

\section{Funding Sources}

Research was performed without any funding.

\section{Author Contributions}

K.S., N.H., and U.B.-P. performed the study design, analyzed the specimens, and wrote the paper and A.V. and W.S. carefully reviewed the manuscript. 


\section{References}

1 Orfanos CE, Happle R, editors. Hair and Hair Diseases. Berlin: Springer; 1990; pp. 111-13.

2 Chuang YH, Dean D, Allen J, Dawber R, Wojnarowska F. Comparison between the expression of basement membrane zone antigens of human interfollicular epidermis and anagen hair follicle using indirect immunofluorescence. Br J Dermatol. 2003 Aug;149(2): 274-81.

3 Horne KA, Jahoda CA. Restoration of hair growth by surgical implantation of follicular dermal sheath. Development. 1992 Nov; 116(3):563-71

4 Weber L, Krieg T, Müller PK, Kirsch E, Timpl R. Immunofluorescent localization of type IV collagen and laminin in human skin and its application in junctional zone pathology. $\mathrm{Br} \mathrm{J}$ Dermatol. 1982 Mar;106(3):267-73.

5 Camacho F, Montagna W. Trichology. Diseases of the pilosebaceous follicle. Madrid: W. Libros Princeps/Bibliotheca Aula Medica; 1997. pp. 19-22.

6 Pinkus F. Anatomie der Haut. In: Jadassohn J, editor. Handbuch der Haut- und Geschlechtskrankheiten. Berlin: Julius Springer; 1927. pp. 147-249.

7 Guesdon JL, Ternynck T, Avrameas S. The use of avidin-biotin interaction in immunoenzymatic techniques. J Histochem Cytochem. 1979 Aug;27(8):1131-9.

8 Schadendorf D, Tiedemann KH, Haas N, Czarnetzki BM. Detection of human papillomaviruses in paraffin-embedded Condylomata acuminata - comparison of immunohistochemistry, in situ hybridization, and polymerase chain reaction. J Invest Dermatol. 1991 Sep;97(3):549-54.
9 Pinkus F. Über Hautsinnesorgane neben dem menschlichen Haar (Haarscheibe) und ihre vergleichend-anatomische Bedeutung. Arch Mikrobiol Anat Entwickl Gesch. 1904;65: 121-79.

10 Orfanos CE. Haar und Haarkrankheiten. 2nd ed. Stuttgart: Gustav Fischer Verlag; 1991.

11 Braun-Falco O. Dynamics of normal and pathological hair growth. Arch Klin Exp Dermatol. 1966;227(1):419-52. German.

12 Messenger AG, Elliott K, Westgate GE, Gibson WT. Distribution of extracellular matrix molecules in human hair follicles. Ann N Y Acad Sci. 1991 Dec;642(1):253-62.

13 Cotsarelis G, Sun TT, Lavker RM. Label-retaining cells reside in the bulge area of pilosebaceous unit: implications for follicular stem cells, hair cycle, and skin carcinogenesis. Cell. 1990 Jun;61(7):1329-37.

14 Sun TT, Cotsarelis G, Lavker RM. Hair follicular stem cells: the bulge-activation hypothesis. J Invest Dermatol. 1991 May;96(5): $77 \mathrm{~S}-8 \mathrm{~S}$.

15 Wollina U. Histochemistry of the human hair follicle with consideration of anagen phases I to VI. Acta Histochem. 1992;92(2):171-8

16 Jahoda CA, Mauger A, Bard S, Sengel P. Changes in fibronectin, laminin and type IV collagen distribution relate to basement membrane restructuring during the rat vibrissa follicle hair growth cycle. J Anat. 1992 Aug;181(Pt 1):47-60.

17 Jih DM, Lyle S, Elenitsas R, Elder DE, Cotsarelis G. Cytokeratin 15 expression in trichoepitheliomas and a subset of basal cell carcinomas suggests they originate from hair follicle stem cells. J Cutan Pathol. 1999 Mar; 26(3):113-8.
18 Lyle S, Christofidou-Solomidou M, Liu Y, Elder DE, Albelda S, Cotsarelis G. The C8/144B monoclonal antibody recognizes cytokeratin 15 and defines the location of human hair follicle stem cells. J Cell Sci. 1998 Nov;111(Pt 21):3179-88.

19 Horak ER, Leek R, Klenk N, LeJeune S, Smith $\mathrm{K}$, Stuart N, et al. Angiogenesis, assessed by platelet/endothelial cell adhesion molecule antibodies, as indicator of node metastases and survival in breast cancer. Lancet. 1992 Nov;340(8828):1120-4.

20 Mecklenburg L, Tobin DJ, Müller-Röver S, Handjiski B, Wendt G, Peters EM, et al. Active hair growth (anagen) is associated with angiogenesis. J Invest Dermatol. 2000 May;114(5): 909-16.

21 Poblet E, Jimenez-Acosta F, Rocamora A. QBEND/10 (anti-CD34 antibody) in external root sheath cells and follicular tumors. $\mathrm{J} \mathrm{Cu}-$ tan Pathol. 1994 Jun;21(3):224-8.

22 Trempus CS, Morris RJ, Bortner CD, Cotsarelis G, Faircloth RS, Reece JM, et al. Enrichment for living murine keratinocytes from the hair follicle bulge with the cell surface marker CD34. J Invest Dermatol. 2003 Apr;120(4): 501-11.

23 Botchkarev VA, Eichmüller S, Johansson O, Paus R. Hair cycle-dependent plasticity of skin and hair follicle innervation in normal murine skin. J Comp Neurol. 1997 Sep; 386(3):379-95.

24 Gho CG, Braun JE, Tilli CM, Neumann HA, Ramaekers FC. Human follicular stem cells: their presence in plucked hair and follicular cell culture. Br J Dermatol. 2004 May;150(5): 860-8. 